\title{
Development of specific care strategies to maintain and recover among survivors' health after Kumamoto Earthquake in 2016-intervention program to prevent PTSD and depression
}

\author{
Shiori USAMI ${ }^{1 *}$, NoriakiTakahashi ${ }^{2}$ and Ryoma Kayano \\ ${ }^{1}$ Faculty of Life Sciences, Kumamoto University, Japan \\ ${ }^{2}$ Musashigaoka-Clinic, Japan \\ ${ }^{3}$ Technical Officer, WHO Centre for Health Development, Kobe
}

Increasing scale and frequency of disasters has indicated the priority to mitigate disaster risk and impacts. Considering the disaster risk management for health, special attention and relevant strategy for vulnerable population is required. Older population has physical, cognitive and mental vulnerability such as multiple chronic diseases which can be worsen by the hazardous events, and/or high rate acquisition of post-traumatic stress disorder (PTSD) and depression after disaster.

The purpose of this study is to evaluate the effects of a therapeutic intervention program in order to prevent the worsening of Post Traumatic Stress Response(PTSR) and Depressive conditions that aggravate chronic illness (diabetes, Liver disease, problems with alcohol and others) that tend to occur after disaster. This intervention program is to improve self-care abilities.

People who were affected by the 2016 Kumamoto earthquake in areas where there was major damage. The subject of the research will be about 200 people with informed consent for this research. This program is implemented Two-day program in every month after Kumamoto earthquake. This therapeutic program was developed by Dr. Hidefumi Kotani, a leading expert on trauma treatment after disasters. This program was based on the experience of the Great East Japan Earthquake, and validity of the program was secured by the research. Day 1 program is to carry out psychoeducation for the purpose of preventing the worsening of PTSR and depressive conditions (two hours) and to implement open consultations and individual consultations on confused feelings related to loss of motivation for life and work, distress and the decline of self-care (conduct for 30 minutes per person). Day 2 program is psychodynamic, small-group psychotherapy in three groups (eight people per group) with each session lasting three hours. It has been done by Dr. Hidefumi Kotani, Institute of Psychoanalytic Systems Psyochotherapy in Tokyo, and Shiori Usami, Certified Nurse Specialist in Japan. During the first hour, the group will be made into a psychologically safe space in which people can feel safe speaking and will touch on the earthquakes and their work after the earthquakes, and the exhaustion, pain and sadness from the changes in their lives. In the group's psychologically safe space, the functions of the individual's self will be triggered in the next hour, changing anger and sadness to love, while touching on the feelings of anger and sadness. The energies of anger and love will also be transformed into the individual's energy, and the individual will be made to confront and acknowledge his or her loss of motivation and depression. Furthermore, the group will touch on the fear of death that emerged with the earthquakes and people will express their own confused feelings, concerns and worries over changes in their lives and work after the earthquakes. They will listen to what others say, confirm their own present situation and confirm that they are not the only ones who are experiencing feelings of fear. In the last hour, people will once again review their needs for their own self-care, examine their work, lives, relations with other people and the rebuilding of their lives, improve their self-care abilities to take care of themselves while overcoming the loss of motivation, fear and concerns that they are experiencing after the earthquakes, and practice self-care actions. In the mid- to long-term, the self-care abilities they acquired, and their self-care actions will be able to prevent the worsening of chronic illnesses.

Data collection for program evaluation will be done in four times by using PTSD evaluation sheet (Primary care PTSD sheet), a depressive state evaluation sheet (CES-D, The Centre for Epidemiologic Studies Depression Scale), a sheet of dynamic change for trauma response (developed by Dr. Kotani) and SF-8 (HRQOL, Health Related Quality of Life) as a self-care evaluation on health conditions. These data is analyzed by quantitatively and qualitatively. Now it is found that through this intervention, PTSR, CES-D, DCTR and SF-8 are significant difference between pre-intervention and post-intervention. Furthermore through qualitative analysis we will make clear the intervention techniques to promote self-care to prevent PTSD and

${ }^{\star}$ Correspondence to: Shiori USAMI, Kumamoto University, Faculty of life sciences, kumamoto university, 4-24-1, kuhonji, chuo-ku, kumamoto city, Japan, Tel: 0963735470; E-mail: susami@kumamoto-u.ac.jp

Key words: disasters, post-traumatic stress disorder (PTSD), self-care

Received: May 14, 2018; Accepted: May 18, 2018; Published: May 21, 2018 
USAMI S (2018) Development of specific care strategies to maintain and recover among survivors' health after Kumamoto Earthquake in 2016-intervention program to prevent PTSD and depression

depression after disaster. In the future CNS or Nurse Practitioner (NP) as APN (Advanced Practice of Nurse) will implement this program after disaster by using this intervention technique. Now based on this research, we have developed PAS-SCT (Psychoanalytic Systems Self Care Therapy, Kotani \& Usami,2018) which APN, nurse, and nurse administrator can make use of.

\section{Funding}

This research is funded by WHO Centre for Health Development (WHO Kobe Centre; WKC, Principle Investigator of this research, Dr. Aiko Yamamoto) and this research is one part of "Development of Specific Care Strategies to Maintain and Recover among Survivors' Health after disasters".

Copyright: $\odot 2018$ USAMI S. This is an open-access article distributed under the terms of the Creative Commons Attribution License, which permits unrestricted use, distribution, and reproduction in any medium, provided the original author and source are credited. 